\title{
Study of deuteron-proton charge exchange reaction at small transfer momentum
}

\author{
N.B.Ladygina ${ }^{1}$ A.V.Shebeko ${ }^{2}$ \\ ${ }^{1}$ Laboratory of High Energies, Joint Institute for Nuclear Research, 141980 Dubna, Russia, \\ ${ }^{2}$ NSC Kharkov Institute of Physics 83 Technology, 61108 Kharkov, Ukraine.
}

\begin{abstract}
The charge exchange reaction $p d \rightarrow n p p$ at $1 \mathrm{GeV}$ projectile proton energy is studied in the multiple-scattering expansion technique. This reaction is considered in a special kinematics, when the transfer momentum from the beam proton to fast neutron is close to zero. The differential cross section and a set of polarization observables are calculated. It was shown that contribution of the final state interaction between two protons is very significant.
\end{abstract}

\section{Introduction}

Within the several last decades the deuteron- proton charge exchange reaction has been studied both from experimental and theoretical point of view. A considerable interest to this reaction is connected, first of all, with an opportunity to extract some information about spin-dependent part of the elementary nucleon-nucleon charge exchange amplitudes. This idea was suggested by Pomeranchuk [1] already in 1951, but up to now it is actual. Later this supposition has been developed in $[2,3,4]$. It was shown, that in the plane-wave impulse approximation (PWIA) the differential cross section and tensor analyzing power $T_{20}$ in the dp-charge exchange reaction are actually fully determined by the spin-dependent part of the elementary $n p \rightarrow p n$ amplitudes. The analogous result was obtained in [5], where this process has been studied in the Bethe-Salpeter formalism.

The differential cross section of the $d p \rightarrow n p p$ reaction at $3.34 \mathrm{GeV} / \mathrm{c}$ deuteron beam has been measured in 1970's in the 1m hydrogen bubble chamber of the JINR Synchrophasatron $[6,7]$. However, the obtained statistics is not sufficient to evaluate the magnitude of the spin-dependent part of the elementary amplitude. Nowadays the experiment on the study of the dp-charge exchange reaction at the small transfer momentum in GeV-region is planned at ANKE setup at COSY [8]. The aim of this experiment is to provide the information about the spin-dependent np-elastic scattering amplitudes in the energy region, where the phase-shift analysis data are absent.

From our point of view, under kinematical conditions proposed in this experiment, when momentum of the emitted neutron has the same direction and magnitude as the beam proton (in the deuteron rest frame), and relative momentum of two protons is very small, the final state interaction (FSI) effects play very important role. The contribution of the D-wave in the DWF into differential cross section in this kinematics must be negligible [9]. However, for the polarization observables the influence of the D-component can be significant.

The goal of our paper is to study the importance of the D-wave and FSI effects under kinematical conditions of the planned experiment. We consider $p d \rightarrow n p p$ reaction in 
the approach, which has been used by us to describe the pd breakup process at $1 \mathrm{GeV}$ projectile proton energy [10]. This approach is based on the Alt-Grassberger-Sandhas formulation of the multiple-scattering theory for the three-nucleon system. The matrix inversion method $[11,12]$ has been applied to take account of the FSI contributions. Since unpolarized and polarized mode of the deuteron beam are supposed to be employed in the experiment, we also calculate both the differential cross section and a set of the polarization observables. It should be noted, in this paper we have not considered the Coulomb interaction in the (pp)-pair. This problem is nontrivial and requires a special investigation.

The paper is organized as following. In sect.2 the short description of the general theoretical formalism is given. The special kinematics, when the transfer momentum from the beam proton to neutron is close to zero, is considered in sect.3. The results of our calculations for the differential cross section and polarization observables are presented in sect.4. The figures in this section demonstrate the behaviour of this observables obtained in the PWIA and PWIA+FSI with D-wave in the DWF included and without it. The significant dependence of the calculation results on the elementary $\mathrm{NN}$-amplitudes is also shown. We conclude with sect.5.

\section{Theoretical Formalism}

In accordance to the three-body collision theory let us write the matrix element of the deuteron proton charge exchange reaction

$$
p(\vec{p})+d(\overrightarrow{0}) \rightarrow n\left(\vec{p}_{1}\right)+p\left(\vec{p}_{2}\right)+p\left(\vec{p}_{3}\right)
$$

in the following form $[10]$

$$
U_{p d \rightarrow n p p}=\sqrt{2}<123\left|[1-(2,3)]\left[1+t_{23}\left(E-E_{1}\right) g_{23}\left(E-E_{1}\right)\right] t_{12}^{\text {sym }}\right| 1(23)>,
$$

where the operator $g_{23}\left(E-E_{1}\right)$ is a free propagator for the (23)-subsystem and the scattering operator $t_{23}\left(E-E_{1}\right)$ satisfies the Lippmann-Schwinger (LS) equation with two-body force operator $V_{23}$ as driving term

$$
t_{23}\left(E-E_{1}\right)=V_{23}+V_{23} g_{23}\left(E-E_{1}\right) t_{23}\left(E-E_{1}\right) .
$$

Here $E$ is the total energy of the three-nucleon system $E=E_{1}+E_{2}+E_{3}$.

Let us rewrite the matrix element (2) indicating explicitly the particle quantum numbers,

$$
U_{p d \rightarrow n p p}=\sqrt{2}<\overrightarrow{p_{1}} m_{1} \tau_{1}, \overrightarrow{p_{2}} m_{2} \tau_{2}, \overrightarrow{p_{3}} m_{3} \tau_{3}\left|[1-(2,3)] \omega_{23} t_{12}^{s y m}\right| \overrightarrow{p m} \tau, \psi_{1 M_{d} 00}(23)>,
$$

where $\omega_{23}=\left[1+t_{23}\left(E-E_{1}\right) g_{23}\left(E-E_{1}\right)\right]$ and the the spin and isospin projections denoted as $m$ and $\tau$, respectively. The permutation operator for two nucleons $(i, j)$ was introduced

here. The operator $t_{12}^{\text {sym }}$ is symmetrized NN-operator, $t_{12}^{\text {sym }}=[1-(1,2)] t_{12}$. Inserting the unity

$$
\mathbf{1}=\int d \vec{p}^{\prime}\left|\vec{p}^{\prime} m^{\prime} \tau^{\prime}><\vec{p}^{\prime} m^{\prime} \tau^{\prime}\right|
$$


we get the following expression for the reaction amplitude

$$
\begin{aligned}
\mathcal{J}= & (-1)^{1 / 2+\tau_{3}^{\prime}}<\frac{1}{2} \tau_{2} \frac{1}{2} \tau_{3}\left|T \tau_{2}+\tau_{3}><\frac{1}{2} \tau_{2}^{\prime} \frac{1}{2} \tau_{3}^{\prime}\right| T \tau_{2}+\tau_{3}><\frac{1}{2} \tau_{1} \frac{1}{2} \tau_{2}^{\prime} \mid T^{\prime} M_{T^{\prime}}> \\
& <\frac{1}{2} \tau \frac{1}{2}-\tau_{3}^{\prime}\left|T^{\prime} M_{T^{\prime}}><\frac{1}{2} m_{2} \frac{1}{2} m_{3}\right| S M_{S}><\frac{1}{2} m_{2}^{\prime} \frac{1}{2} m_{3}^{\prime} \mid S M_{S}^{\prime}> \\
& \int d \vec{p}_{0}^{\prime}\left\langle\vec{p}_{0}, S M_{S}\left|1+m_{N} \frac{t^{S T}\left(E-E_{1}\right)}{{\overrightarrow{p_{0}}}^{2}-\vec{p}_{0}^{\prime 2}+i 0}\right|{\overrightarrow{p_{0}}}^{\prime}, S M_{S}^{\prime}\right\rangle \\
& <\overrightarrow{p_{1}} m_{1},\left({\overrightarrow{p_{0}}}^{\prime}+\vec{q} / 2\right) m_{2}^{\prime}\left|t_{s y m}^{T^{\prime}}\left(E-E_{3}^{\prime}\right)\right| \overrightarrow{p m},\left(\vec{p}_{0}^{\prime}-\vec{q} / 2\right) m^{\prime \prime}> \\
& <m^{\prime \prime} m_{3}^{\prime} \mid \psi_{1 M_{d}}\left(\vec{p}_{0}{ }^{\prime}-\vec{q} / 2\right)>-(2 \leftrightarrow 3),
\end{aligned}
$$

where $E_{3}^{\prime}=\sqrt{m_{N}^{2}+\left(\vec{p}_{0}^{\prime}-\vec{q} / 2\right)^{2}}, m_{N}$ the nucleon mass, and we have introduced the momentum transfer $\vec{q}=\vec{p}-\overrightarrow{p_{1}}$, relative momenta $\vec{p}_{0}=\frac{1}{2}\left(\overrightarrow{p_{2}}-\overrightarrow{p_{3}}\right)$ and ${\overrightarrow{p_{0}}}^{\prime}=\frac{1}{2}\left({\overrightarrow{p_{2}}}^{\prime}-{\overrightarrow{p_{3}}}^{\prime}\right)$. Henceforth, all summations over dummy discrete indices are implied.

In momentum representation the DWF $\psi_{1 M_{d}}(\vec{k})$ with spin projection $M_{d}$ is written as

$$
\left|\psi_{1 M_{d}}(\vec{k})>=\sum_{L=0,2} \sum_{M_{L}=-L}^{L}<L M_{L} 1 M_{s}\right| 1 M_{d}>u_{L}(k) Y_{L}^{M_{L}}(\hat{k}) \mid 1 M_{s}>,
$$

with the spherical harmonics $Y_{L}^{M_{L}}(\hat{k})$ and the Clebsh-Gordon coefficients in standard form. In our calculations, we have employed the following parameterizations of the Sand D- state wave functions

$$
u_{0}(p)=\sqrt{\frac{2}{\pi}} \sum_{i} \frac{C_{i}}{\alpha_{i}^{2}+p^{2}} \quad, \quad u_{2}(p)=\sqrt{\frac{2}{\pi}} \sum_{i} \frac{D_{i}}{\beta_{i}^{2}+p^{2}}
$$

as proposed in refs. $[13,14,15]$.

We assume that $\tau=\tau_{2}=\tau_{3}=1 / 2$ and $\tau_{1}=-1 / 2$. Then the isotopic coefficient can be calculated and Eq.(4) is simplified

$$
\begin{aligned}
\mathcal{J}= & \frac{1}{2}<\frac{1}{2} m_{2} \frac{1}{2} m_{3}\left|S M_{S}><\frac{1}{2} m_{2}^{\prime} \frac{1}{2} m_{3}^{\prime}\right| S M_{S}^{\prime}> \\
& <L M_{L} 1 \mathcal{M}_{\mathcal{S}}\left|1 M_{d}><\frac{1}{2} m^{\prime \prime} \frac{1}{2} m_{3}^{\prime}\right| 1 \mathcal{M}_{\mathcal{S}}> \\
& \int d{\overrightarrow{p_{0}}}^{\prime}<\psi_{\vec{p}_{0} S M_{S} T M_{T}}^{(-)} \mid \vec{p}_{0}^{\prime} S M_{S}^{\prime} T M_{T}>u_{L}\left(\left|\vec{p}_{0}^{\prime}-\vec{q} / 2\right|\right) Y_{L}^{M_{L}}\left(\overrightarrow{p_{0}^{\prime}} \widehat{-\vec{q} / 2)}\right. \\
& <\overrightarrow{p_{1}} m_{1},\left({\overrightarrow{p_{0}}}^{\prime}+\vec{q} / 2\right) m_{2}^{\prime}\left|t_{s y m}^{0}\left(E-E_{3}^{\prime}\right)-t_{s y m}^{1}\left(E-E_{3}^{\prime}\right)\right| \overrightarrow{p m},\left(\vec{p}_{0}^{\prime}-\vec{q} / 2\right) m^{\prime \prime}> \\
& \quad-(2 \leftrightarrow 3),
\end{aligned}
$$

The wave function of the final p-p pair

$$
\begin{array}{r}
<\psi_{\overrightarrow{p_{0}} S M_{S} T M_{T}}^{(-)} \mid \vec{p}_{0}{ }^{\prime} S M_{S}^{\prime} T M_{T}>=\delta\left(\overrightarrow{p_{0}}-\overrightarrow{p_{0}}\right) \delta_{M_{s} M_{s}^{\prime}}+ \\
\frac{m_{N}}{{\overrightarrow{p_{0}}}^{2}-{\overrightarrow{p_{0}}}^{2}+i 0}<\overrightarrow{p_{0}} S M_{S}\left|t^{S T}\right|{\overrightarrow{p_{0}}}^{\prime} S M_{S}^{\prime}>
\end{array}
$$

contains the FSI part, which can be taken in different ways.

In this paper we use the matrix inversion method (MIM) suggested in refs. $[11,12]$ and applied to study the deuteron electro-disintegration $[16,17]$ and deuteron proton breakup 
process [10]. As in ref. [16], we consider the truncated partial-wave expansion,

$$
\begin{aligned}
& <\psi_{\vec{p}_{0} S M_{S} T M_{T}}^{(-)} \mid \vec{p}_{0}{ }^{\prime} S M_{S}^{\prime} T M_{T}>=\delta_{M_{S} M_{S}^{\prime}} \delta\left(\vec{p}_{0}-{\overrightarrow{p_{0}}}^{\prime}\right)+ \\
& \sum_{J=0}^{J_{\max }} \sum_{M_{J}=-J}^{J} Y_{l}^{\mu}\left(\hat{p}_{0}\right)<l \mu S M_{S}\left|J M_{J}>\psi_{l l^{\prime}}^{\alpha}\left(p_{0}{ }^{\prime}\right)<l^{\prime} \mu^{\prime} S M_{S}^{\prime}\right| J M_{J}>Y_{l^{\prime}}^{* \mu^{\prime}}\left(\hat{p}_{0}{ }^{\prime}\right),
\end{aligned}
$$

where $J_{\max }$ is the maximum value of the total angular momentum in p-p partial waves and $\alpha=\{J, S, T\}$ is the set of conserved quantum numbers. The radial functions $\psi_{l l^{\prime}}^{\alpha}\left(p_{0}{ }^{\prime}\right)$ are related via

$$
\psi_{l l^{\prime}}^{\alpha}\left(p_{0}{ }^{\prime}\right)=\sum_{l^{\prime \prime}} O_{l l^{\prime \prime}} \varphi_{l^{\prime \prime} l^{\prime}}^{\alpha}\left(p_{0}^{\prime}\right)-\frac{\delta\left(p_{0}^{\prime}-p_{0}\right)}{p_{0}^{2}} \delta_{l l^{\prime}}
$$

to the partial wave functions $\varphi_{l^{\prime \prime} l^{\prime}}^{\alpha}\left(p_{0}^{\prime}\right)$, which have the asymptotics of standing waves. The coefficients $O_{l l^{\prime \prime}}$ can be expressed in terms of the corresponding phase shifts and mixing parameters [16].

Within the MIM, the functions $\varphi_{l l^{\prime}}^{\alpha}\left(p_{0}^{\prime}\right)$ can be represented as

$$
\varphi_{l l^{\prime}}^{\alpha}\left(p_{0}{ }^{\prime}\right)=\sum_{j=1}^{N+1} B_{l l^{\prime}}^{\alpha}(j) \frac{\delta\left(p_{0}^{\prime}-p_{j}\right)}{p_{j}^{2}}
$$

where the coefficients $B_{l l^{\prime}}^{\alpha}(j)$ fulfill a set of linear algebraic equations approximately equivalent to the $L S$ integral equation for the $p-p$ scattering problem ${ }^{1}$. Here $N$ is the dimension of this set, $p_{j}$ are the grid points associated with the Gaussian nodes over the interval $[-1,1]$ and $p_{N+1}=p_{0}$ (details can be found in ref. [18]). It should be noted that in this way the nucleon wave function is expressed by a series of $\delta$-functions allowing one to reduce a triple integral in Eq. (7) to a double one. In addition, the method offers the opportunity to consider the nucleon wave function in the continuum directly in momentum space what simplifies all subsequent calculations.

\section{Collinear geometry}

In this paper we consider the special kinematics, when transfer momentum $\vec{q}=\vec{p}-\vec{p}_{1}$ is close to zero. In other words, the neutron momentum has the same value and direction as the beam proton. In fact, since the difference between proton and neutron masses and deuteron binding energy take place, the transfer momentum is not exactly zero, $q \approx 1.8$ $\mathrm{MeV} / \mathrm{c}$. But because of this value is very small and has no significant influence on the results, we shall suppose $q=0$ in the subsequent calculations.

Under such kinematical conditions one can neglect the dependence of the high-energy nucleon-nucleon matrix $t_{N N}\left(E-E_{3}^{\prime}\right)$ in Eq.(7) on the internal nucleon-nucleon momentum in the deuteron and express it in the center-of-mass system (c.m.s.) through three independent amplitudes

$$
t_{N N}^{c m}(\vec{q}=0)=A+(F-B)\left(\vec{\sigma}_{1} \hat{Q}^{*}\right)\left(\vec{\sigma}_{2} \hat{Q}^{*}\right)+B\left(\vec{\sigma}_{1} \vec{\sigma}_{2}\right)
$$

\footnotetext{
${ }^{1}$ We neglect here the Coulomb interaction in the (pp)-pair.
} 
where

$$
\hat{Q}^{*}=\frac{\overrightarrow{p^{*}+\vec{p}^{\prime}}}{\left|\overrightarrow{p^{*}}+\overrightarrow{p^{\prime}}\right|}=\hat{p}^{*}
$$

with the fast proton (neutron) momentum $\vec{p}^{*}$. We use some results of relativistic potential theory $[19,20]$ to relate this NN t-matrix in the c.m.s. with that in the frame of interest (see, also [10]).

$$
\begin{aligned}
<m_{1} m_{2}^{\prime}, \vec{p} \vec{p}_{0}^{\prime}|t| \overrightarrow{p p}_{0}^{\prime}, m m^{\prime \prime}>= & N N^{\prime} F<m_{1}\left|D^{\dagger}(\vec{u}, \vec{p})\right| \mu_{1}><m_{2}^{\prime}\left|D^{\dagger}\left(\vec{u}, \vec{p}_{0}^{\prime}\right)\right| \mu_{2}^{\prime}>\quad(13) \\
& <\mu_{1} \mu_{2}^{\prime}\left|t_{c m}\left(\vec{p}^{*}\right)\right| \mu \mu^{\prime \prime}><\mu|D(\vec{u}, \vec{p})| m><\mu^{\prime \prime}\left|D\left(\vec{u}, \vec{p}_{0}^{\prime}\right)\right| m^{\prime \prime}>,
\end{aligned}
$$

where $D$ is a Wigner rotation operator in the spin space and $u$ is a four-velocity. In our kinematical situation, when $\vec{p}=\vec{p}_{1} \gg \vec{p}_{0}^{\prime}$, each of these operators is slightly different from the unit operator, so that in a good approximation the $t_{N N}$-matrix in the frame of interest has the same spin structure. The product of the normalization factors $N$ and $N^{\prime}$ and kinematical factor $F$ is

$$
N N^{\prime} F=\frac{m_{N}+E_{p}}{4 E_{p}},
$$

In such a way, we have following relation for high-energy NN t-matrix in the different frames of reference

$$
<m_{1} m_{2}^{\prime}\left|t\left(\vec{p}, \vec{p}_{0}^{\prime}\right)\right| m m^{\prime \prime}>=\frac{m_{N}+E_{p}}{4 E_{p}}<m_{1} m_{2}^{\prime}\left|t_{c m}\left(\vec{p}^{*}\right)\right| m m^{\prime \prime}>
$$

To evaluate such quantities without their momentum angular decomposition we use the phenomenological model suggested by Love and Franey in refs. [21]. In this approach the corresponding matrix elements are expressed through the effective NN-interaction operators sandwiched between the initial and final plane-wave states, that enables us to extend this construction to the off-shell case. Obviously, such off-shell extrapolation does not change the general spin structure.

Since the (pp)-pair belongs to the isotriplet, one can anticipate that the FSI in the ${ }^{1} S_{0}$ state is prevalent at comparatively small $p_{0}$-values. In such a way we get the following expression for amplitude of the dp charge exchange process

$$
\begin{aligned}
\mathcal{J}= & \mathcal{J}_{P W I A}+\mathcal{J}_{{ }^{1} S_{0}} \\
\mathcal{J}_{P W I A}= & \frac{m_{N}+E_{p}}{4 E_{p}}<L M_{L} 1 \mathcal{M}_{\mathcal{S}} \mid 1 M_{D}>u_{L}\left(p_{0}^{\prime}\right) Y_{L}^{M_{L}}\left(\widehat{p_{0}^{\prime}}\right) \\
& \left\{<\frac{1}{2} m_{2}^{\prime} \frac{1}{2} m_{3}\left|1 \mathcal{M}_{\mathcal{S}}><m_{1} m_{2}\right| t_{c m}^{0}\left(\vec{p}^{*}\right)-t_{c m}^{1}\left(\vec{p}^{*}\right) \mid m m_{2}^{\prime}>-\right. \\
& \left.<\frac{1}{2} m_{2}^{\prime} \frac{1}{2} m_{2}\left|1 \mathcal{M}_{\mathcal{S}}><m_{1} m_{3}\right| t_{c m}^{0}\left(\vec{p}^{*}\right)-t_{c m}^{1}\left(\vec{p}^{*}\right) \mid m m_{2}^{\prime}>\right\} \\
\mathcal{J}_{S_{0}}= & \frac{(-1)^{1-m_{2}-m_{2}^{\prime}}}{4 \pi} \frac{m_{N}+E_{p}}{4 E_{p}}<L M_{L} 1 \mathcal{M}_{\mathcal{S}}\left|1 M_{D}>\delta_{m_{2}-m_{3}}<\frac{1}{2} m^{\prime \prime} \frac{1}{2}-m_{2}^{\prime}\right| 1 \mathcal{M}_{\mathcal{S}}> \\
& \int d p_{0}^{\prime} p_{0}{ }^{2} \int d \hat{p}_{0}{ }^{\prime} u_{L}\left(p_{0}^{\prime}\right) Y_{L}^{M_{L}}\left(\widehat{p_{0}^{\prime}}\right) \psi_{00}^{001}\left(p_{0}^{\prime}\right) \\
& <m_{1} m_{2}^{\prime}\left|t_{c m}^{0}\left(\vec{p}^{*}\right)-t_{c m}^{1}\left(\vec{p}^{*}\right)\right| m m^{\prime \prime}>
\end{aligned}
$$


One can see, we have very simple integral over angular variables $\hat{p}_{0}^{\prime}$. As the result of this integration we get following relation for $\mathcal{J}_{1} S_{0}$

$$
\begin{aligned}
\mathcal{J}_{1_{S_{0}}}= & \frac{(-1)^{1-m_{2}-m_{2}^{\prime}}}{\sqrt{4 \pi}} \frac{m_{N}+E_{p}}{4 E_{p}} \delta_{m_{2}-m_{3}}<\frac{1}{2} m^{\prime \prime} \frac{1}{2}-m_{2}^{\prime} \mid 1 M_{D}> \\
& <m_{1} m_{2}^{\prime}\left|t_{c m}^{0}\left(\vec{p}^{*}\right)-t_{c m}^{1}\left(\vec{p}^{*}\right)\right| m m^{\prime \prime}>\int d p_{0}{ }^{\prime}{p_{0}}^{\prime 2} \psi_{00}^{001}\left(p_{0}^{\prime}\right) u_{0}\left(p_{0}^{\prime}\right)
\end{aligned}
$$

Note, the integral over radial variable $p_{0}^{\prime}$ also has no any difficulties, since $\psi_{00}^{001}\left(p_{0}^{\prime}\right)$ contains $\delta$-functions.

\section{Results and discussions}

We define unpolarized $2 \rightarrow 3$ cross section by the standard manner

$$
\sigma(d p \rightarrow n p p)=(2 \pi)^{4} \frac{E_{p}}{p} \int d \overrightarrow{p_{1}} d \overrightarrow{p_{2}} d \overrightarrow{p_{3}}|\overline{\mathcal{J}}|^{2} \delta^{4}(4-\text { momentum })
$$

where $|\overline{\mathcal{J}}|^{2}=1 / 6 \operatorname{Tr}\left(\mathcal{J J}^{+}\right)$is the square of the process amplitude averaged over all particles spin states. Using the $\delta^{3}$-function to eliminate the $\vec{p}_{3}$ integration and changing variables from $\vec{p}_{1}$ to $\vec{q}$ we have

$\sigma(d p \rightarrow n p p)=(2 \pi)^{4} \frac{E_{p}}{p} \int d \vec{q} d \overrightarrow{p_{2}}|\overline{\mathcal{J}}|^{2} \delta\left(m_{d}+E_{p}-\sqrt{m_{N}^{2}+(\vec{p}-\vec{q})^{2}}-E_{2}-\sqrt{m_{N}^{2}+\left(\vec{q}-\vec{p}_{2}\right)^{2}}\right)$.

Taking $p_{2}, q \ll p$ this expression can be reduced to

$$
\sigma(d p \rightarrow n p p)=(2 \pi)^{6} \frac{E_{p}^{2}}{2 p^{2}} \int d q^{2} d p_{2} d \cos \theta_{2} p_{2}^{2}|\overline{\mathcal{J}}|^{2}
$$

We define general spin observable related with polarization of initial particles in terms of the Pauli $2 \times 2$ spin matrices $\sigma$ for the proton and a set of spin operators $S$ for deuteron $[22]$ as following

$$
C_{\alpha \beta}=\frac{\operatorname{Tr}\left(\mathcal{J} \sigma_{\alpha} S_{\beta} \mathcal{J}\right)}{\operatorname{Tr}\left(\mathcal{J} \mathcal{J}^{+}\right)}
$$

where indices $\alpha$ and $\beta$ refer to the proton and deuteron polarization, respectively; $\sigma_{0}$ and $S_{0}$ corresponding to the unpolarized particles are the unit matrices of two and three dimensions. In such a way, Eqs.(16-17) for dp- charge exchange amplitude enables us to get the relation for any variable of this process taking into account two slow protons final state interaction in ${ }^{1} S_{0}$-state. So, we have following expression for the spin- averaged squared amplitude

$$
\begin{aligned}
C_{0} \equiv & \operatorname{Tr}\left(\mathcal{J J}^{+}\right)=\frac{1}{4 \pi}\left(\frac{m_{N}+E_{p}}{2 E_{p}}\right)^{2}\left\{2\left(2 B^{2}+F^{2}\right)\left(\mathcal{U}^{2}\left(p_{2}\right)+w^{2}\left(p_{2}\right)\right)+\right. \\
& \left.\left(F^{2}-B^{2}\right) w\left(p_{2}\right)\left(w\left(p_{2}\right)-2 \sqrt{2} \operatorname{Re} \mathcal{U}\left(p_{2}\right)\right)\left(3 \cos ^{2} \theta_{2}-1\right)\right\}
\end{aligned}
$$

where $\mathcal{U}\left(p_{2}\right)=u\left(p_{2}\right)+\int d p_{0}^{\prime} p_{0}^{\prime 2} \psi_{00}^{001}\left(p_{0}^{\prime}\right) u\left(p_{0}^{\prime}\right)$ is the S-component of the DWF corrected on the FSI of the (pp)-pair. One can see, the $C_{0}$ is independent on the neutron and proton 
azimuthal angles $\phi_{q}$ and $\phi_{2}$. To obtain Eq.(20) we have considered this fact and performed the integration over azimuthal angles. We use a right-hand coordinate system defined in accordance to the Madison convention [23]. The quantization $z$-axis is along the beam proton momentum $\vec{p}$. Since the direction of $\vec{p} \times \vec{p}_{1}$ is undefined in the collinear geometry, we choose the $y$-axis normal to the beam momentum. Then third axis is $\vec{x}=\vec{y} \times \vec{z}$.

The tensor analyzing power can be presented in the following form

$$
\begin{aligned}
C_{0, y y} \cdot C_{0}= & \frac{1}{2} \frac{1}{4 \pi}\left(\frac{m_{N}+E_{p}}{2 E_{p}}\right)^{2}\left\{4\left(F^{2}-B^{2}\right)\left(\mathcal{U}^{2}\left(p_{2}\right)+w^{2}\left(p_{2}\right)\right)+\right. \\
& \left(2 F^{2}+B^{2}\right) w\left(p_{2}\right)\left(w\left(p_{2}\right)-2 \sqrt{2} \operatorname{Re} \mathcal{U}\left(p_{2}\right)\right)\left(3 \cos ^{2} \theta_{2}-1\right)+ \\
& 9 B^{2} w\left(p_{2}\right)\left(w\left(p_{2}\right)-2 \sqrt{2} \operatorname{Re} \mathcal{U}\left(p_{2}\right)\right) \sin ^{2} \theta_{2} \cos 2 \phi_{2}- \\
& \left.54\left(F^{2}-B^{2}\right) w^{2}\left(p_{2}\right) \sin ^{2} \theta_{2} \cos ^{2} \theta_{2} \sin ^{2} \phi_{2}\right\}
\end{aligned}
$$

Note, that only squared nucleon- nucleon spin- flip amplitudes $B^{2}$ and $F^{2}$ are in expression for the tensor analyzing power $C_{0, y y}$ and differential cross section. However, the spin correlation due to vector polarization of deuteron and beam proton contains the interference terms of this amplitudes

$$
\begin{aligned}
C_{y, y} \cdot C_{0}= & -\frac{2}{4 \pi}\left(\frac{m_{N}+E_{p}}{2 E_{p}}\right)^{2} \operatorname{Re}\left(F B^{*}\right)\left\{2 \mathcal{U}^{2}\left(p_{2}\right)-w^{2}\left(p_{2}\right)-\right. \\
& \left.w\left(p_{2}\right)\left(w\left(p_{2}\right)+\sqrt{2} \operatorname{Re} \mathcal{U}\left(p_{2}\right)\right)\left(1-3 \sin ^{2} \theta_{2} \sin ^{2} \phi_{2}\right)\right\}+ \\
& 12 w\left(p_{2}\right) \operatorname{Im}\left(F B^{*}\right) \operatorname{Im\mathcal {U}}\left(p_{2}\right)\left(\cos ^{2} \theta_{2}-\sin ^{2} \theta_{2} \cos ^{2} \phi_{2}\right)
\end{aligned}
$$

It is interesting, that there is the term proportional to the imaginary part of $\mathcal{U}\left(p_{2}\right)$. It has a non-zero value only in case when FSI is taken into account. The analogous result we have obtained for the vector-tensor spin correlation

$$
\begin{aligned}
C_{y, x z} \cdot C_{0}= & -\frac{3}{4 \pi}\left(\frac{m_{N}+E_{p}}{2 E_{p}}\right)^{2} \operatorname{Im}\left(F B^{*}\right)\left\{2 \mathcal{U}^{2}\left(p_{2}\right)-w^{2}\left(p_{2}\right)-\right. \\
& w\left(p_{2}\right)\left(w\left(p_{2}\right)+\sqrt{2} \operatorname{Re} \mathcal{U}\left(p_{2}\right)\right)\left(1-3 \sin ^{2} \theta_{2} \sin ^{2} \phi_{2}\right)+ \\
& \left.18 w^{2}\left(p_{2}\right) \sin ^{2} \theta_{2} \cos ^{2} \theta_{2} \cos ^{2} \phi_{2}\right\}- \\
& 3 \sqrt{2} w\left(p_{2}\right) \operatorname{Re}\left(F B^{*}\right) \operatorname{Im\mathcal {U}}\left(p_{2}\right)\left(\cos ^{2} \theta_{2}-\sin ^{2} \theta_{2} \cos ^{2} \phi_{2}\right)
\end{aligned}
$$

In order to evaluate these observables we consider kinematics, when one of the slow protons is emitted along the beam direction as well as neutron, i.e. $\theta_{2}=0^{0}$. Under such conditions Eqs. (22)-(25) are significantly simplified.

$$
\begin{aligned}
C_{0}= & \frac{1}{2 \pi}\left(\frac{m_{N}+E_{p}}{2 E_{p}}\right)^{2}\left\{\left(2 B^{2}+F^{2}\right)\left(\mathcal{U}^{2}\left(p_{2}\right)+w^{2}\left(p_{2}\right)\right)+\right. \\
& \left.\left(F^{2}-B^{2}\right) w\left(p_{2}\right)\left(w\left(p_{2}\right)-2 \sqrt{2} \operatorname{Re} \mathcal{U}\left(p_{2}\right)\right)\right\} \\
C_{0, y y} \cdot C_{0}= & \frac{1}{4 \pi}\left(\frac{m_{N}+E_{p}}{2 E_{p}}\right)^{2}\left\{2\left(F^{2}-B^{2}\right)\left(\mathcal{U}^{2}\left(p_{2}\right)+w^{2}\left(p_{2}\right)\right)+\right. \\
& \left.\left(2 F^{2}+B^{2}\right) w\left(p_{2}\right)\left(w\left(p_{2}\right)-2 \sqrt{2} \operatorname{Re} \mathcal{U}\left(p_{2}\right)\right)\right\}
\end{aligned}
$$




$$
\begin{aligned}
C_{y, y} \cdot C_{0}= & -\frac{2}{4 \pi}\left(\frac{m_{N}+E_{p}}{2 E_{p}}\right)^{2}\left\{\operatorname{Re}\left(F B^{*}\right)\left[2 \mathcal{U}^{2}\left(p_{2}\right)-2 w^{2}\left(p_{2}\right)-\sqrt{2} \operatorname{Re} \mathcal{U}\left(p_{2}\right) w\left(p_{2}\right)\right]-\right. \\
& \left.3 \sqrt{2} \operatorname{Im}\left(F B^{*}\right) \operatorname{Im} \mathcal{U}\left(p_{2}\right) w\left(p_{2}\right)\right\} \\
C_{y, x z} \cdot C_{0}= & -\frac{3}{4 \pi}\left(\frac{m_{N}+E_{p}}{2 E_{p}}\right)^{2}\left\{\operatorname{Im}\left(F B^{*}\right)\left[2 \mathcal{U}^{2}\left(p_{2}\right)-2 w^{2}\left(p_{2}\right)-\sqrt{2} \operatorname{Re} \mathcal{U}\left(p_{2}\right) w\left(p_{2}\right)\right]+\right. \\
& \left.3 \sqrt{2} \operatorname{Re}\left(F B^{*}\right) \operatorname{Im} \mathcal{U}\left(p_{2}\right) w\left(p_{2}\right)\right\}
\end{aligned}
$$

The differential cross section and three polarization observables are presented in figs.(14). The Love and Franey parametrization with a set of parameters obtained by fitting of the modern phase shift data SP00 [25, 26] has been employed for NN-amplitude. The full lines correspond to calculations with taking into account both the FSI in the (pp)-pair and S- and D- waves in the deuteron. The results obtained in the PWIA are shown by the dashed lines. The dash-dotted lines in the figs.(2-4) for polarization observables are the full calculation results without D-wave in the DWF. In fig.1 the full and dash-dotted lines are undistinguished. All calculations were carried out with Paris NN-potential [24] and Paris DWF [13].

One can see, the FSI contribution to the differential cross section (fig.1) is significant even at the very small proton momentum, while for the polarization observables the difference between PWIA and PWIA +FSI is visible only for $p_{2} \geq 10-15 \mathrm{MeV} / \mathrm{c}$. However, with increase of the proton momentum up to $50 \mathrm{MeV} / \mathrm{c}$ the importance of the FSI corrections to the PWIA also increases.

Note, the absolute value of the tensor analyzing power $C_{0, y y}$ (fig.2) in the momentum interval of interest is near zero. In order to understand the source of that, we disregard the D-wave in the DWF. Then, as a consequence from Eq.(26), the polarization observables are defined by the ratio of the nucleon-nucleon charge exchange amplitudes only

$$
\begin{aligned}
C_{0, y y} & =\frac{1}{2} \cdot \frac{F^{2}-B^{2}}{2 B^{2}+F^{2}} \\
C_{y, y} & =-2 \cdot \frac{\operatorname{Re}\left(F B^{*}\right)}{2 B^{2}+F^{2}} \\
C_{y, x z} & =-3 \cdot \frac{\operatorname{Im}\left(F B^{*}\right)}{2 B^{2}+F^{2}}
\end{aligned}
$$

Thus, the nearness of the tensor analyzing power to zero indicates that the absolute values of the spin-flip NN-amplitudes approximately equal each other, $|B| \approx|F|$.

The vector-tensor spin correlation $C_{y, x z}$ (fig.4) has also very small value, $\left|C_{y, x z}\right| \approx 0.06$. The magnitude of this observable decreases up to zero for $p_{2} \approx 50 \mathrm{MeV} / \mathrm{c}$, if the FSI corrections and D-wave in the deuteron are taken into account, while it is almost constant in the PWIA and PWIA+FSI without D-wave. One can see from Eqs. $(26,27)$ for $C_{y, x z}$, the reason of this behaviour is connected with the small value of the imaginary part of the nucleon-nucleon amplitudes product, $\operatorname{Im}\left(F B^{*}\right)$. In such a way, the great contribution into $C_{y, x z}$ gives the term proportional to $\operatorname{Re}\left(F B^{*}\right)$, which defined by D-wave and imaginary part of the generalized function $\mathcal{U}\left(p_{2}\right)$. Note, that $\operatorname{Im\mathcal {U}}\left(p_{2}\right) \neq 0$, if FSI taken into account.

The other situation is for the vector-vector spin correlation $C_{y, y}$ (fig.3). The term proportional to $\operatorname{Re}\left(F B^{*}\right)$ gives also a considerable contribution in this observable, but it is multiplied on the $\mathcal{U}^{2}\left(p_{2}\right)$. The magnitude of $C_{y, y}$ is close to the theoretical limit 
$-2 / 3$, that confirms to the conclusion about approximate equality of the nucleon-nucleon amplitudes, $|B|$ and $|F|$. Besides, this allows to conclude, that the relative phase between these amplitudes is close to zero. It is seen from Eq.(27), where D-wave was neglected.

Since all the considered observables are defined by the elementary nucleon-nucleon amplitudes mostly, it is interesting to compare their behaviour for different NNparametrization. In figs.(5-8) we present the same observables as in figs.(1-4) for two sets of parameters. The full line corresponds to the parameterization based on the modern shift analysis SP00 [25, 26]. The dashed line is obtained using a set of parameters for NN-amplitude from [21]. One can see, the difference for the differential cross section (fig.5) is about 1.5-2 times. The absolute value for the tensor analyzing power $C_{0, y y}$ (fig.6) with new parametrization is about 2.5 times smaller than that with parametrization [21]. The opposite situation is for the vector-vector spin correlation $C_{y, y}$ (fig.7), where new predictions is 1.5 times lager in comparison with old parametrization of the NN-amplitude [21]. The predictions of these two parameterizations for the vector- tensor spin correlation $C_{y, x z}$ (fig.8) are even in opposite sign. Nevertheless, the qualitative behaviour of the curves in figs. (6-8) for different sets of parameters is similar.

\section{Conclusion}

We have studied the deuteron -proton charge exchange reaction at $1 \mathrm{GeV}$ energy in special kinematics, $\vec{q} \approx 0$. The influence of the D-wave in the deuteron and FSI between two slow protons has been considered. It was shown, that D-wave and FSI effects are negligible for the polarization observables at the proton momentum up to 10-15 MeV/c. As the result, in this region the polarization observables are defined by the ratio of the nucleon- nucleon charge exchange amplitudes only. However, it should not be ignored, that importance of the D-wave and, especially, FSI into polarization observables increases at $p_{2} \geq 15$ $\mathrm{MeV} / \mathrm{c}$. In such a way, we conclude, that ratio of the nucleon- nucleon charge exchange amplitudes and phase shift between their can be extracted from experimental data rather simple, if the experimental conditions and technical setup possibilities allow to work in this small momentum interval. In opposite case, this procedure is more complicated and model dependent. It should be remembered, that FSI contribution into the differential cross section is very significant in comparison with PWIA predictions even at very small proton momentum. This fact does not enables us to get the absolute value of the nucleonnucleon spin flip amplitudes without considering the FSI corrections.

We are grateful to V.V.Glagolev, M.S.Nioradze and A.Kacharava for inspiration of interest to this problem. The authors are thankful to V.P. Ladygin for fruitful discussions.

\section{References}

[1] I.Pomeranchuk, Doklady Academii Nauk USSR 78, 249 (1951)

[2] N.W.Dean, Phys.Rev. D5, 1661 (1972); Phys.Rev. D5, 2832 (1972) 
[3] D.V.Bugg, C.Wilkin, Nucl.Phys. A467, 575 (1987)

[4] J.Carbonell, M.B.Barbaro, C.Wilkin, Nucl.Phys. A529, 653 (1991)

[5] L.P.Kaptari, B.Kampfer, S.S.Semikh, S.M.Dorkin, Eur.Phys.J. A17, 119 (2003)

[6] B.Aladashvili et al., Nucl.Phys. B86, 461 (1975)

[7] B.Aladashvili et al., J.Phys. G3, 1225 (1977)

[8] A.Kacharava, F.Rathmann (spokespersons) et al., COSY proposal \# 125, 2003

[9] V.V.Glagolev et al. Eur.Phys.J. A15, 471 (2002)

[10] N.B.Ladygina, A.V.Shebeko, Few Body Syst.33, 49 (2003)

[11] M.I.Haftel, F.Tabakin, Nucl.Phys. A158, 1 (1970)

[12] G.E.Brown, A.D.Jackson, T.T.S.Kuo, Nucl.Phys. A133, 481 (1969)

[13] M. Lacombe et al., Phys.Lett.B 101, 139 (1981)

[14] R.Machleidt, K.Holinde, C.Elster, Phys. Rep. 149, 1 (1987).

[15] R.Machleidt, Phys.Rev. C63, 024001 (2001)

[16] A.Yu.Korchin, Yu.P.Mel'nik, A.V.Shebeko, Few-Body Syst. 9, 211 (1990)

[17] Yu.P.Mel'nik, A.V.Shebeko, Few-Body Syst. 13, 59 (1992)

[18] A.Yu.Korchin, A.V.Shebeko, Preprint KFTI 77-35, Kharkov 1977

[19] L.Heller, G.E.Bohannon, F.Tabakin, Phys. Rev. C13, 742 (1976)

[20] H.Garcilazo, Phys. Rev. C16, 1996 (1976)

[21] W.G.Love, M.A.Franey, Phys.Rev. C24, 1073 (1981); W.G.Love, M.A.Franey, Phys.Rev. C31, 488 (1985)

[22] G.O. Ohlsen, Rep. Prog. Phys. 35, 717 (1972)

[23] Proceedings of the 3-d Int.Symp., Madison,1970 edited by H.H. Barshall, W.Haeberli (Madison, WI: University of Wisconsin Press)

[24] M.Lacombe et al., Phys.Rev. C21, 861 (1980)

[25] R.A.Arndt, I.I.Strakovsky, R.L.Workman, Phys.Rev. C62, 034005 (2000)

[26] http://gwdac.phys.gwu.edu 


\section{Figure captions}

Fig.1 The differential cross section at $\vec{q}=0$ as a function of one of the slow proton momentum. The dashed and full line correspond to the PWIA and PWIA+FSI, respectively.

Fig.2 The tensor analyzing power $C_{y y}$ vs. $p_{2}$. The dashed line corresponds to PWIA; dash-dotted and full lines are PWIA+FSI without D-component in the DWF and with it, respectively.

Fig.3 The spin-correlation $C_{y, y}$ due to the vector polarization of the deuteron. The curves are the same as in fig.2.

Fig.4 The spin-correlation $C_{y, x z}$ due to the tensor polarization of the deuteron. The curves are the same as in fig. 2 .

Fig.5 The differential cross section at $\vec{q}=0$ as a function of $p_{2}$. The dashed and full line correspond to the full calculation with a set of NN-amplitude parameters taken from [21] and fit of SP00 data [26].

Fig.6 The tensor analyzing power $C_{y y}$ vs. $p_{2}$. The curves are the same as in fig.5.

Fig.7 The spin-correlation $C_{y, y}$ due to the vector polarization of the deuteron. The curves are the same as in fig. 5 .

Fig.8 The spin-correlation $C_{y, x z}$ due to the tensor polarization of the deuteron. The curves are the same as in fig.5. 


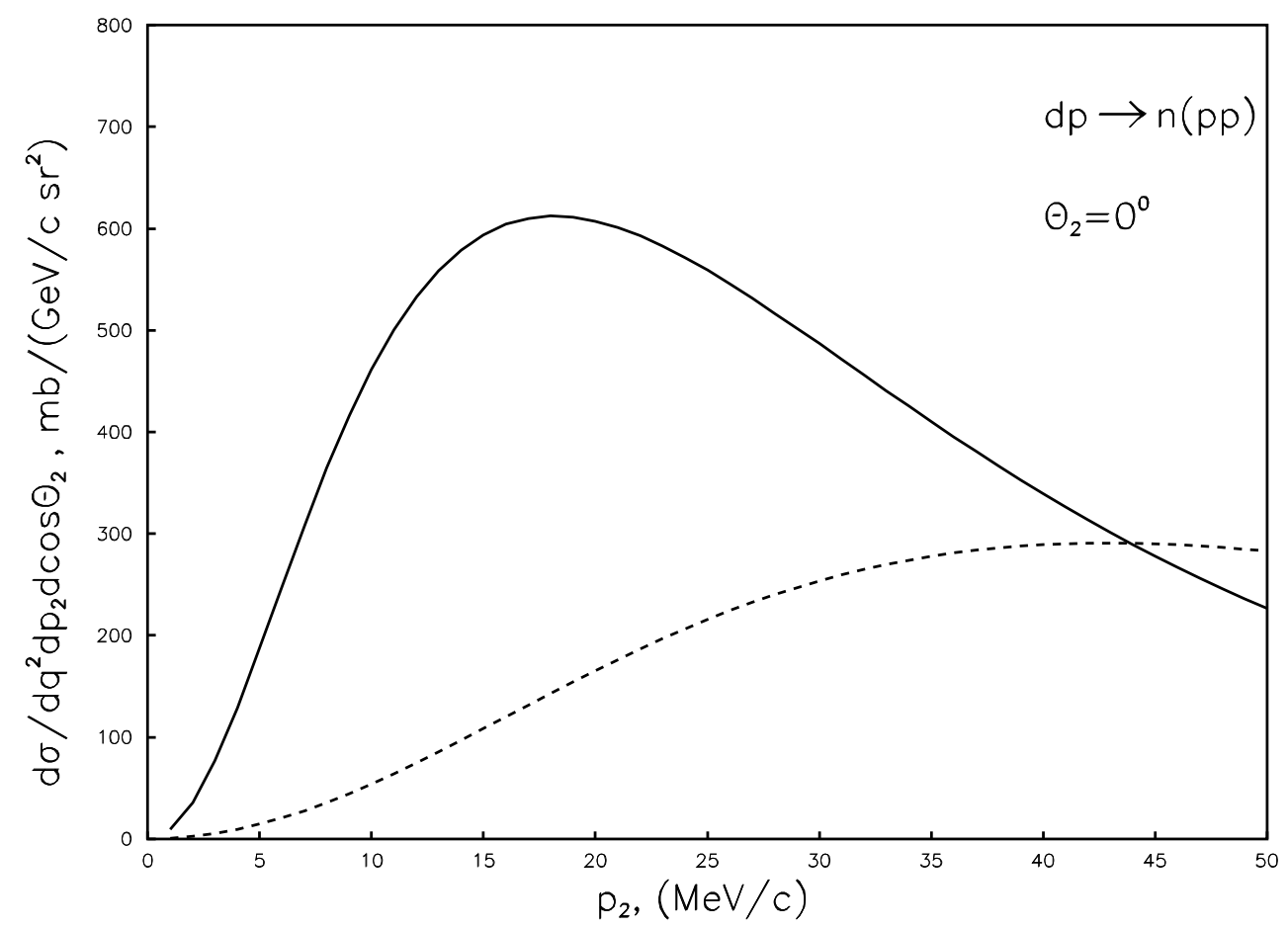

Fig.1 


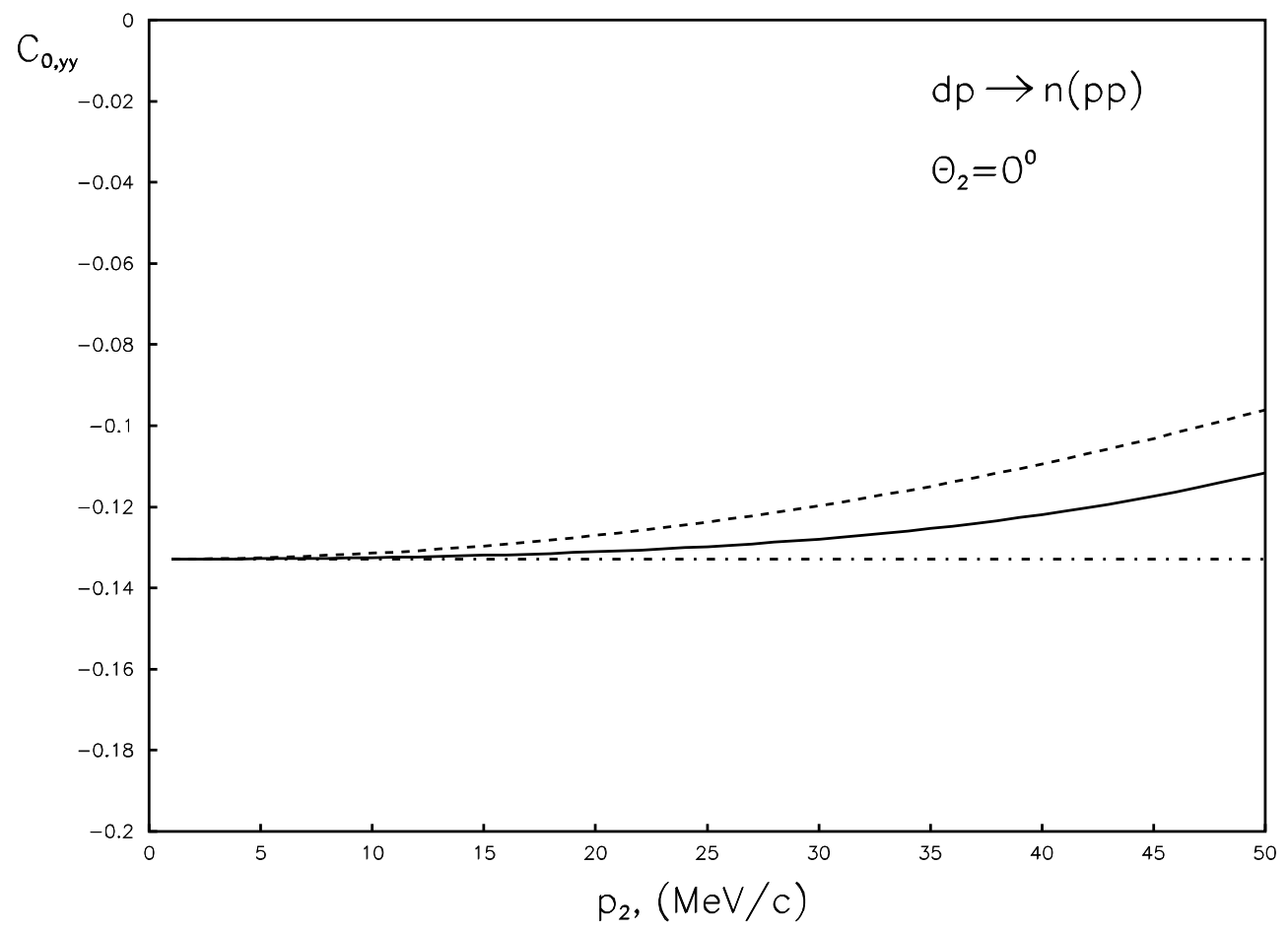

Fig.2 


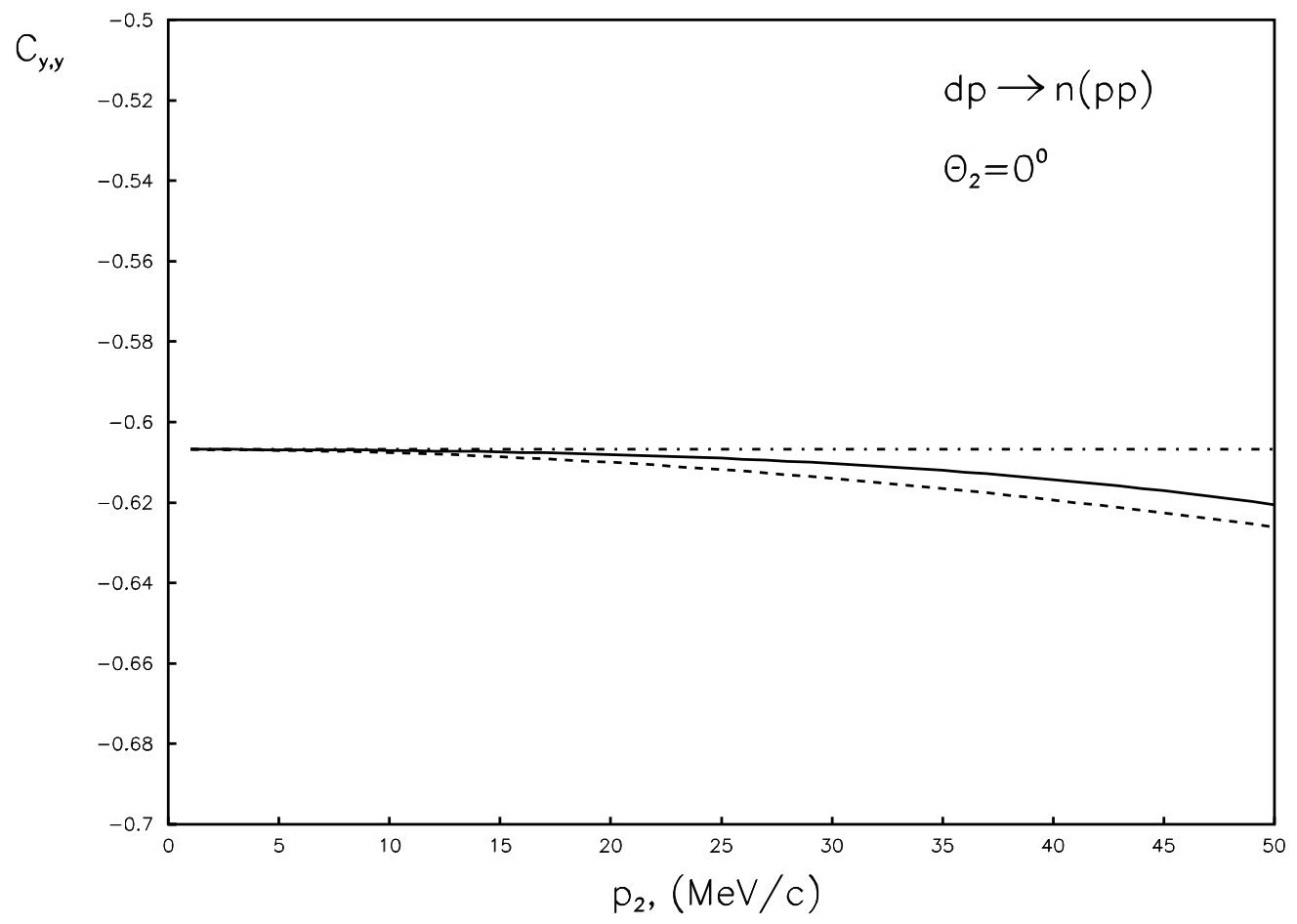

Fig.3 


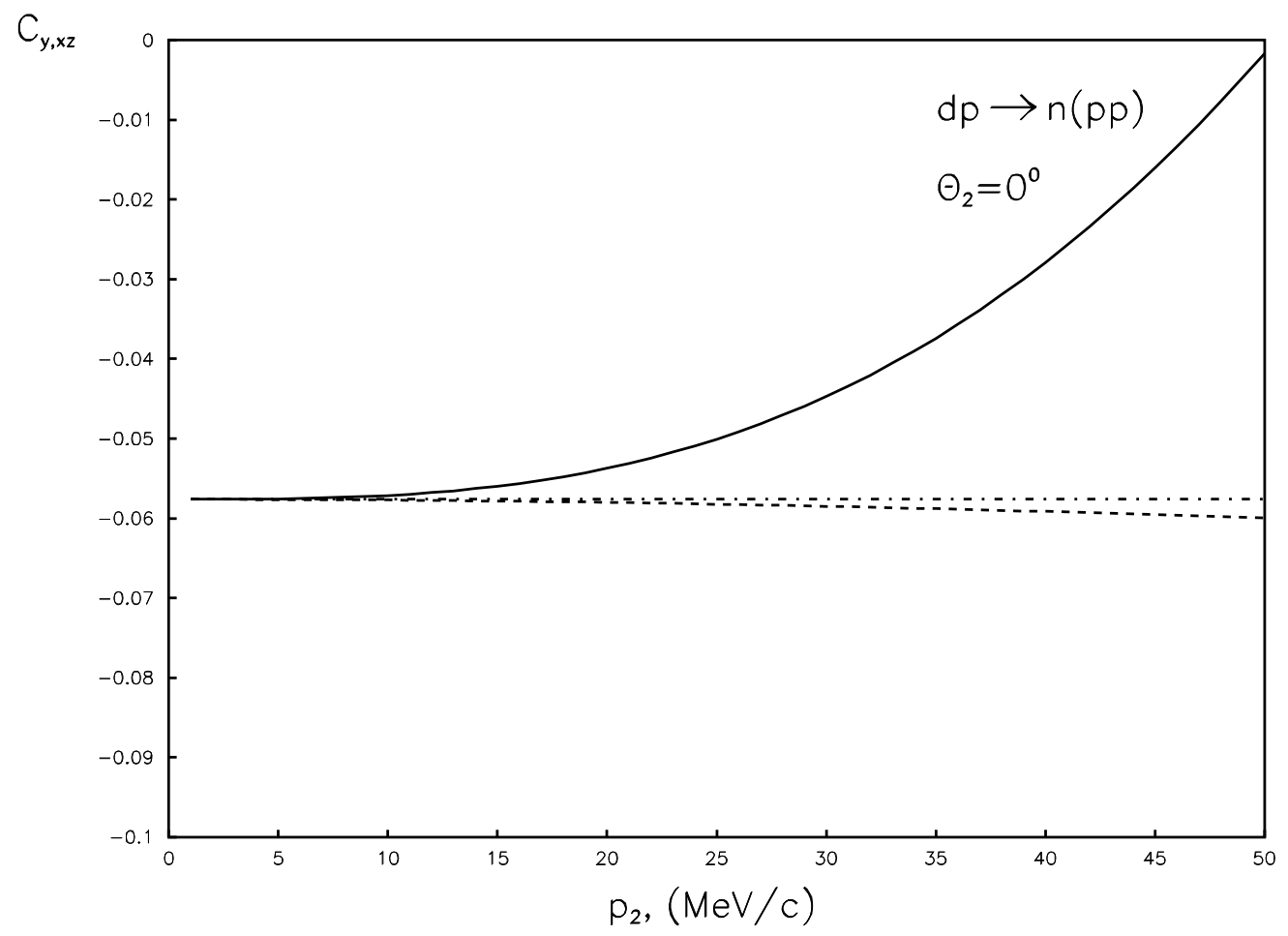

Fig.4 


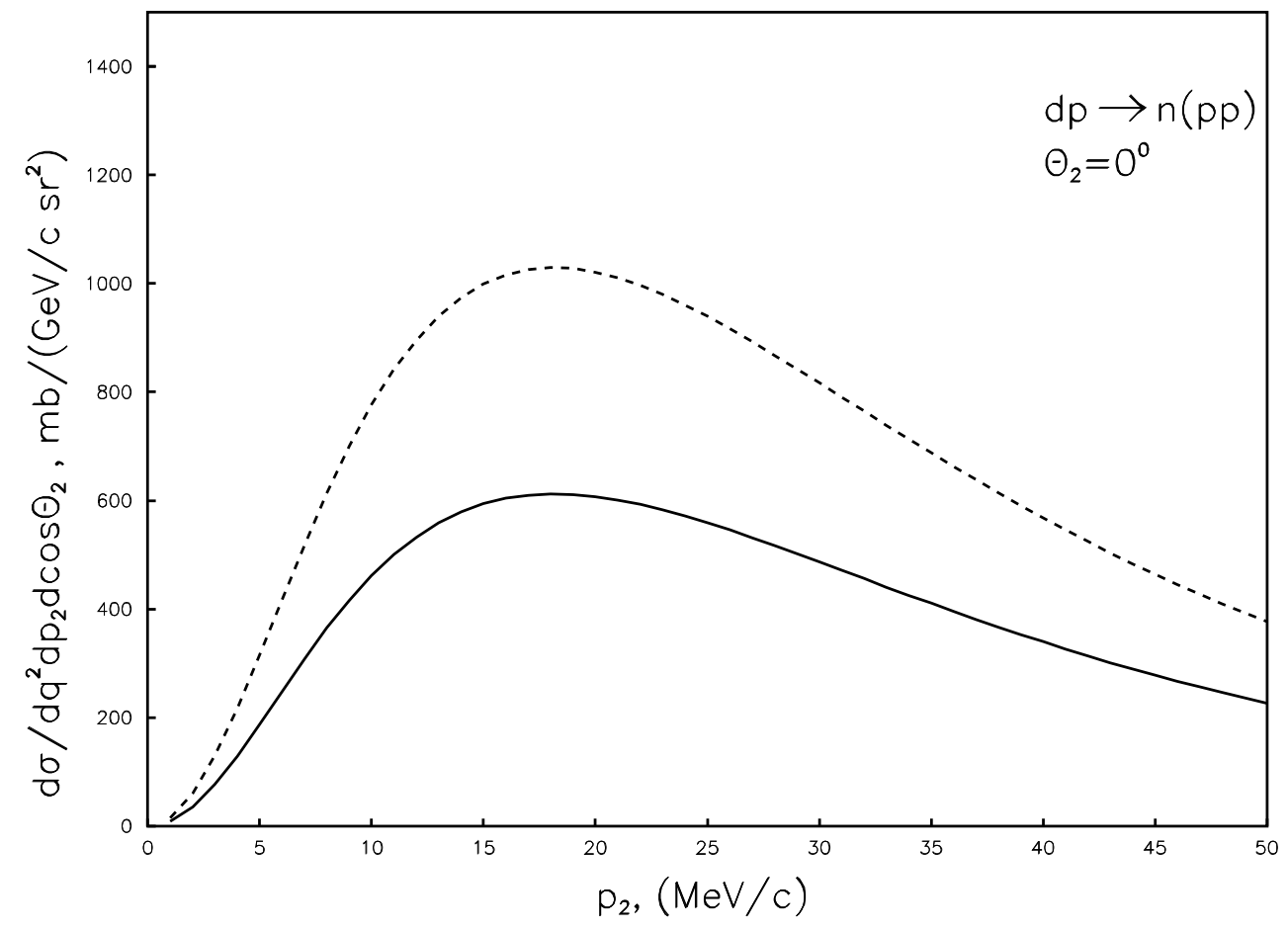

Fig.5 


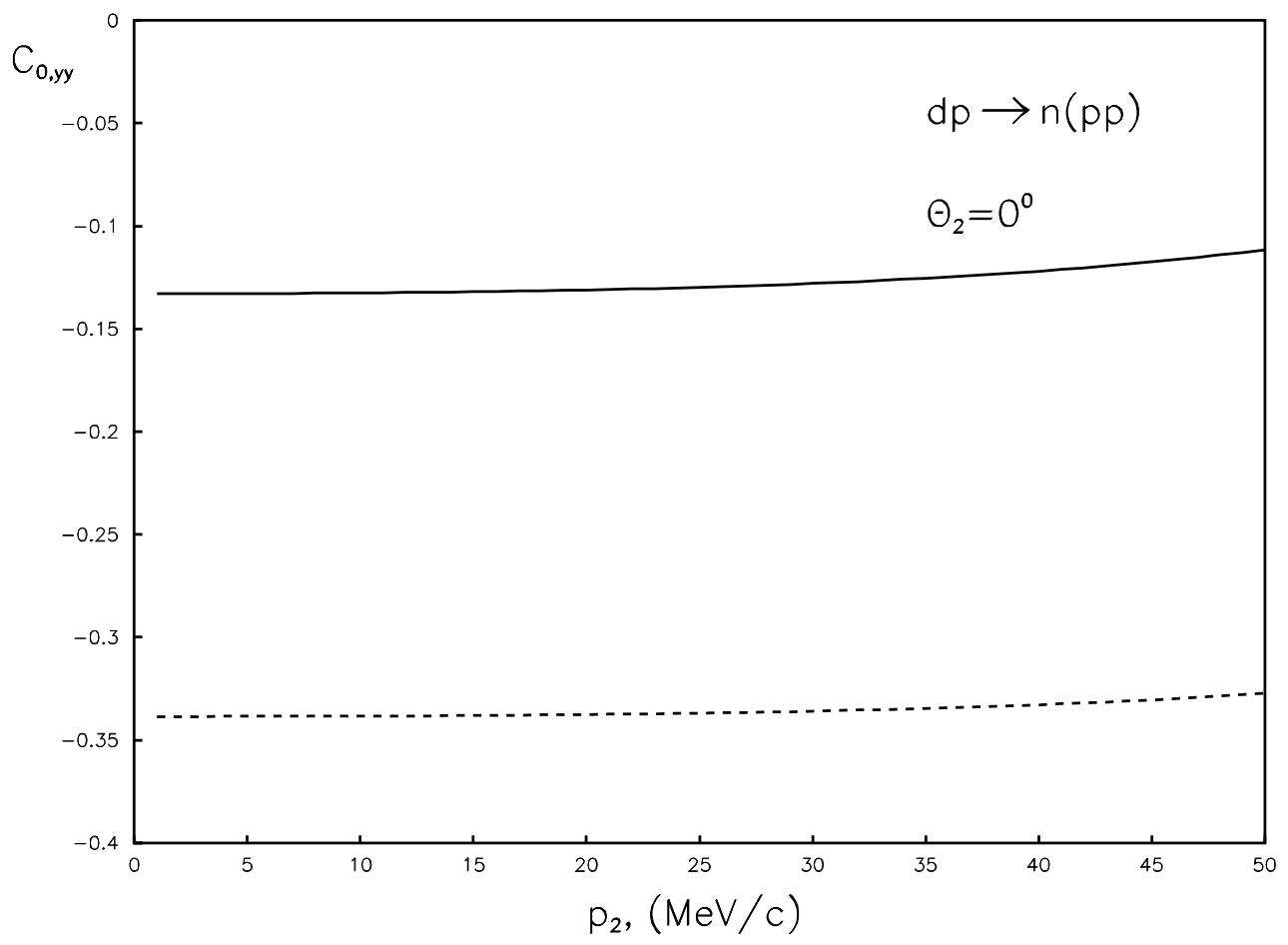

Fig.6 


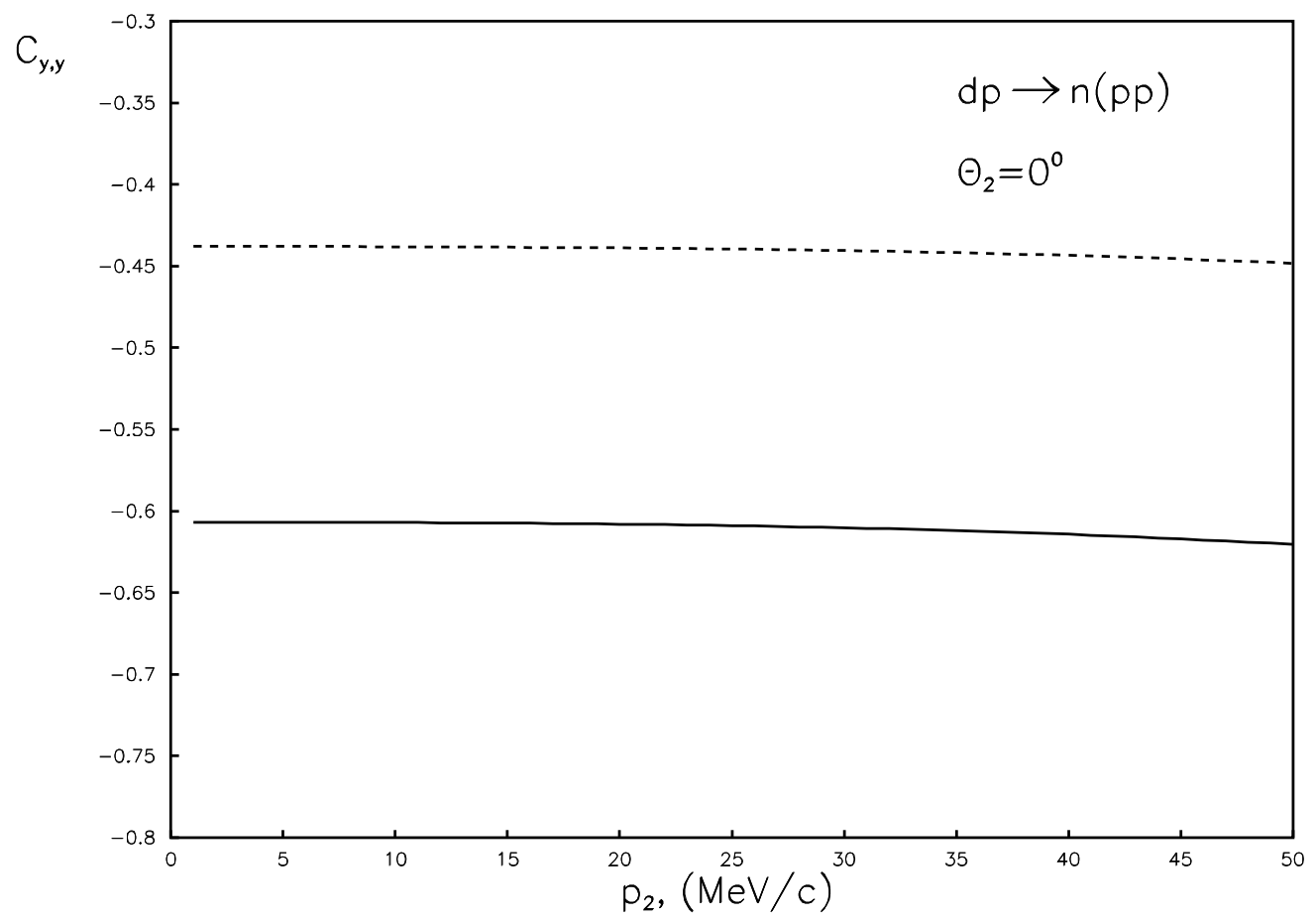

Fig. 7 


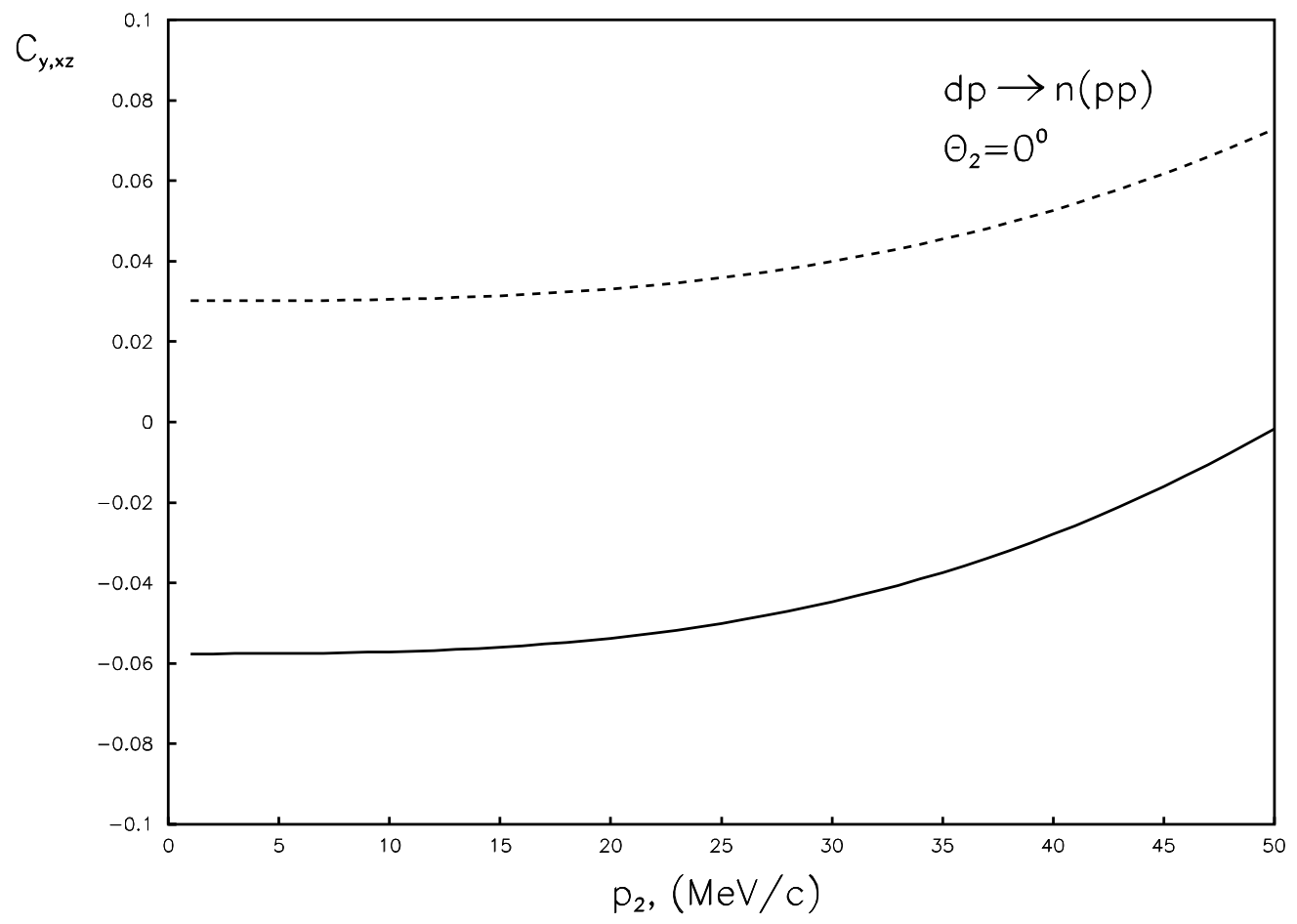

Fig.8 